\title{
The natural yield curve: its concept and measurement
}

\author{
Kei Imakubo* \\ kei.imakubo@boj.or.jp \\ Haruki Kojima* \\ haruki.kojima@boj.or.jp \\ Jouchi Nakajima* \\ jouchi.nakajima@boj.or.jp
}

Papers in the Bank of Japan Working Paper Series are circulated in order to stimulate discussion and comments. Views expressed are those of authors and do not necessarily reflect those of the Bank.

If you have any comment or question on the working paper series, please contact each author.

When making a copy or reproduction of the content for commercial purposes, please contact the Public Relations Department (post.prd8@boj.or.jp) at the Bank in advance to request permission. When making a copy or reproduction, the source, Bank of Japan Working Paper Series, should explicitly be credited. 


\title{
The natural yield curve: its concept and measurement
}

\author{
Kei Imakubo, ${ }^{\dagger}$ Haruki Kojima, $^{\ddagger}$ and Jouchi Nakajima ${ }^{\S}$
}

June 2015

\begin{abstract}
This paper illustrates the concept of the natural yield curve and how to measure it. The natural yield curve extends the idea of the natural rate of interest defined at a single maturity to one defined for all maturities. If the actual real yield curve matches the natural yield curve, the output gap will converge to zero. An empirical analysis using data for Japan shows that past monetary easing programs expanded the gap between the actual real yield curve and the natural yield curve mainly for short and medium maturities and led to accommodative financial conditions. By contrast, the quantitative and qualitative monetary easing policy has expanded the gap for long maturities as well as short and medium maturities. The natural yield curve is expected to provide a useful benchmark in the conduct of both conventional monetary policy and unconventional monetary policy aiming to influence the entire yield curve.
\end{abstract}

JEL classification: C32, E43, E52, E58

Keywords: Natural yield curve; Yield curve gap; Natural rate of interest; Interest rate gap; Term structure

We are grateful for helpful comments from the staff of the Bank of Japan. The views expressed herein are those of the authors alone and do not necessarily reflect those of the Bank of Japan.

† Monetary Affairs Department, Bank of Japan (kei.imakubo@boj.or.jp)

‡ Monetary Affairs Department, Bank of Japan (haruki.kojima@boj.or.jp)

$\S$ Monetary Affairs Department, Bank of Japan (jouchi.nakajima@boj.or.jp) 


\section{Introduction}

The natural rate of interest is the real interest rate at which the economy neither accelerates nor decelerates. If the actual real rate of interest is above the natural rate of interest, the interest rate gap will put downward pressure on output, and vice versa. This characteristic makes the natural rate of interest a reference point for central banks in controlling short-term interest rates. Many central bankers and researchers have attempted to estimate the natural rate of interest using various methods (e.g., Laubach and Williams, 2003). ${ }^{1}$ Also, researchers at the Bank of Japan, such as Oda and Muranaga (2003) and Kamada (2009), have estimated the natural rate of interest for Japan. Yet, in several advanced economies it has been impossible in recent years to use the gap between the actual real rate of interest at a specific maturity - such as the overnight rate - and the corresponding natural rate of interest to consistently evaluate the effects of monetary easing. Short-term nominal rates have hit the zero lower bound and there has hence been no room for a further decline. Against this background, the focus of monetary policy by major central banks has shifted from short-term interest rates to the entire yield curve. Information is therefore needed on not only interest rates at a specific maturity but also the entire yield curve to gauge the overall effects of monetary easing. ${ }^{2}$

From this viewpoint, this paper illustrates the concept of the natural yield curve, which is an extension of the conventional natural rate of interest, and discusses how to measure it. The natural yield curve extends the idea of the natural rate of interest defined at a single maturity to one defined for all maturities. Just as the natural rate of interest is defined as the real interest rate at which the economy neither accelerates nor decelerates, so the natural yield curve is defined as the real yield curve at which the economy neither accelerates nor decelerates. If the actual real yield curve matches the natural yield curve, the output gap will converge to zero. On the other hand, if the actual real yield curve lies above the natural yield curve, financial conditions are

\footnotetext{
${ }^{1}$ See, for example, Bomfim (1997), Neiss and Nelson (2003), Brzoza-Brzezina (2003), Cuaresma et al. (2004), King and Low (2014), and Barsky et al. (2014).

2 Justiniano and Pimiceri (2010) argue that economic activity can be affected by not only the overnight interest rate gap at the current time but also future expectations of the interest rate gap.
} 
contractionary, leading to a contraction of a positive output gap (or an expansion of a negative output gap). By contrast, if the actual real yield curve lies below the natural yield curve, financial conditions are accommodative, leading to a contraction of a negative output gap (or an expansion of a positive output gap).

Just as the natural rate of interest can be derived from the IS curve which describes the relationship between the interest rate gap and the output gap, so the natural yield curve can be derived from the IS curve which describes the relationship between the yield curve gap - the gap between the actual real yield curve and the natural yield curve - and the output gap. Concretely, we decompose yield curves into three factors and then formulate the relationship between the gap of each factor from its equilibrium level and the output gap. Unlike Brzoza-Brzezina and Kotłowski (2014), who propose another approach to estimating the natural yield curve, our approach allows us to distinguish the difference in the sensitivity of the economy to various shapes of the yield curve gap as well as the difference in the term structure between the natural yield curve and the actual real yield curve.

The remainder of this paper is structured as follows. Section 2 introduces the concept of the natural yield curve, while Section 3 presents our estimation approach. Section 4 applies the proposed approach to data for Japan and reports developments in the estimated natural yield curve and yield curve gap since the 1990s. Finally, Section 5 concludes.

\section{The natural yield curve model}

\subsection{Model structure}

This section describes our natural yield curve (NYC) model. Our NYC model is based on Laubach and Williams' (2003) model, which is widely used for measuring the natural rate of interest. In their model, the relationship between the interest rate gap and the output gap is given by the following IS curve: 


$$
y_{t}-y_{t}^{*}=b\left(r_{t}-r_{t}^{*}\right) \text {, }
$$

where $y_{t}$ denotes the log of actual output, $y_{t}^{*}$ the log of potential output, $r_{t}$ the actual rate of interest, and $r_{t}^{*}$ the natural rate of interest. ${ }^{3}$ The output gap and the interest rate gap are defined by $x_{t} \equiv y_{t}-y_{t}^{*}$ and $g_{t} \equiv r_{t}-r_{t}^{*}$, respectively. From Equation (1) it is evident that $x_{t}=0$ if $g_{t}=0$. This means that $r_{t}^{*}$ is the real interest rate at which the economy neither accelerates nor decelerates. Using the Kalman filter method, Laubach and Williams (2003) jointly estimate the unobservable variables, $r_{t}^{*}$ and $y_{t}^{*}$, based on the observation equations - the IS curve in Equation (1) and the Phillips curve - and the state equations for $r_{t}^{*}$ and $y_{t}^{*}$.

As mentioned earlier, when there is little room for further lowering short-term nominal rates, information on interest rates at a specific maturity is insufficient to evaluate the effects of monetary easing. To overcome this limitation, we extend the idea of the natural rate of interest defined at a single maturity to one defined for all maturities. Specifically, we rewrite the relationship between the interest rate gap at a single maturity and the output gap described in Equation (1) to represent the relationship between interest rate gaps for all maturities $0<\tau \leq T$ and the output gap as follows:

$$
y_{t}-y_{t}^{*}=b \int_{0}^{T} \phi(\tau)\left(r_{\tau, t}-r_{\tau, t}^{*}\right) d \tau,
$$

where $r_{\tau, t}$ and $r_{\tau, t}^{*}$ are the actual real rate of interest for maturity $\tau$ at time $t$ and the natural rate of interest for maturity $\tau$ at time $t$, respectively. We refer to $\left\{r_{\tau, t}^{*}\right\}_{\tau=0}^{T}$ and $\left\{r_{\tau, t}\right\}_{\tau=0}^{T}$ as the natural yield curve and the actual real yield curve, respectively. $\phi(\tau)$ is the parameter describing the difference in the sensitivity of the output gap, $x_{t}$, to the interest rate gap at each maturity, $g_{\tau, t} \equiv r_{\tau, t}-r_{\tau, t}^{*}$. It is set as $\phi(\tau) \geq 0$ for all $\tau$ and $\int_{0}^{T} \phi(\tau) d \tau \equiv 1$

Both $\left\{r_{\tau, t}^{*}\right\}_{\tau=0}^{T}$ and $\left\{r_{\tau, t}\right\}_{\tau=0}^{T}$ can be decomposed into the three latent factors described in Nelson and Siegel's (1987) model. Conversely, this means that the interest

\footnotetext{
${ }^{3}$ Laubach and Williams' (2003) model includes lagged terms of the output gap and the interest rate gap, which are omitted in this section for simplicity.
} 
rate at any maturity on the yield curve is given by the combination of these three factors:

$$
\begin{aligned}
& r_{\tau, t}^{*}=L_{t}^{*}+S_{t}^{*} \frac{1-e^{-\lambda \tau}}{\lambda \tau}+C_{t}^{*}\left(\frac{1-e^{-\lambda \tau}}{\lambda \tau}-e^{-\lambda \tau}\right), \text { and } \\
& r_{\tau, t}=L_{t}+S_{t} \frac{1-e^{-\lambda \tau}}{\lambda \tau}+C_{t}\left(\frac{1-e^{-\lambda \tau}}{\lambda \tau}-e^{-\lambda \tau}\right),
\end{aligned}
$$

where $\lambda$ is the parameter that governs the decaying rate of the factor loadings in the second and third terms of the right-hand side of Equations (3) and (4). We assume that the natural yield curve and the actual real yield curve have the same $\lambda$.

$L_{t}^{*}, S_{t}^{*}$, and $C_{t}^{*}$ are the components of the natural yield curve and their loadings are plotted in Figure 1. Following Diebold and Li (2006), we define the factors as follows. $L_{t}^{*}$ in the first term of the right-hand side of Equation (3) is the level factor, which defines the level of the natural yield curve and uniformly affects all maturities. If $L_{t}^{*}$ increases, the natural yield curve shifts upward and vice versa. $S_{t}^{*}$ in the second term is the slope factor, which defines the slope of the natural yield curve and corresponds to the term spread of the natural yield curve. $S_{t}^{*}<0$ implies an upward sloping curve, $S_{t}^{*}>0$ a downward sloping curve, and $S_{t}^{*}=0$ a flat curve. $C_{t}^{*}$ in the third term is the curvature factor, which defines the curvature of the natural yield curve. $C_{t}^{*}<0$ implies that the natural yield curve is bending downward and vice versa. Similarly, $L_{t}, S_{t}$, and $C_{t}$ in Equation (4) can be interpreted as the level, slope, and curvature factors of the actual real yield curve.

Subtracting Equation (3) from Equation (4), we obtain

$$
r_{\tau, t}-r_{\tau, t}^{*}=\left(L_{t}-L_{t}^{*}\right)+\left(S_{t}-S_{t}^{*}\right) \frac{1-e^{-\lambda \tau}}{\lambda \tau}+\left(C_{t}-C_{t}^{*}\right)\left(\frac{1-e^{-\lambda \tau}}{\lambda \tau}-e^{-\lambda \tau}\right)
$$

for all $\tau$. The equation shows that the yield curve gap - the gap between the actual real yield curve and the natural yield curve - can be decomposed into three components: the level gap, $L_{t}-L_{t}^{*}$; the slope gap, $S_{t}-S_{t}^{*}$; and the curvature gap, $C_{t}-C_{t}^{*}$.

The three components of the yield curve gap, $L_{t}-L_{t}^{*}, S_{t}-S_{t}^{*}$, and $C_{t}-C_{t}^{*}$, are closely related to monetary policy actions. A graphic illustration of yield curve gaps in 
response to monetary easing is provided in Figure 2. First, $L_{t}-L_{t}^{*}<0$ implies a parallel downward shift of the real yield curve from the natural yield curve, leading to a negative interest rate gap for all maturities. Such a parallel downward shift occurs when the central bank puts downward pressure on the entire yield curve by purchasing long-term government bonds. Second, $S_{t}-S_{t}^{*}<0$ means that short-term real yields decline. This is a typical response to a policy rate cut by the central bank. The slope of the real yield curve becomes steeper as the negative interest rate gap for shorter maturities expands. Third, $C_{t}-C_{t}^{*}<0$ means that medium-term real yields decline relative to the natural yield curve, leading to a negative interest rate gap for medium maturities. This can happen as a result of forward guidance or a commitment by the central bank to keep its policy expansionary and is observed when market participants expect that the low-interest-rate policy will be kept in place for some time to come.

Substituting Equation (5) into Equation (2), we obtain

$$
y_{t}-y_{t}^{*}=b_{L}\left(L_{t}-L_{t}^{*}\right)+b_{S}\left(S_{t}-S_{t}^{*}\right)+b_{C}\left(C_{t}-C_{t}^{*}\right) \text {, }
$$

where

$$
\left\{\begin{array}{l}
\frac{b_{L}}{b}=\int_{0}^{T} \phi(\tau) d \tau=1 \\
\frac{b_{S}}{b}=\int_{0}^{T}\left\{\phi(\tau) \frac{1-e^{-\lambda \tau}}{\lambda \tau}\right\} d \tau \\
\frac{b_{C}}{b}=\int_{0}^{T}\left\{\phi(\tau)\left(\frac{1-e^{-\lambda \tau}}{\lambda \tau}-e^{-\lambda \tau}\right)\right\} d \tau .
\end{array}\right.
$$

Equation (6) is the basic form of our NYC model. Note that the interest rate gap at each maturity, $g_{\tau, t} \equiv r_{\tau, t}-r_{\tau, t}^{*}$, in Equation (2) is replaced by the level gap, $L_{t}-L_{t}^{*}$, the slope gap, $S_{t}-S_{t}^{*}$, and the curvature gap, $C_{t}-C_{t}^{*}$. If $L_{t}-L_{t}^{*}=S_{t}-S_{t}^{*}=C_{t}-C_{t}^{*}=$ 0 holds, which implies $g_{\tau, t}=0$ for any $\tau$, we obtain $x_{t}=0$. As in the case of the natural rate of interest, the natural yield curve is defined as the real yield curve at which the economy neither accelerates nor decelerates.

\subsection{Yield curve gap and interest rate gap}

Equation (7) represents the relationship between the sensitivity of the output gap to each component of the yield curve gap, $b_{L}, b_{S}$, and $b_{C}$, and the sensitivity to the interest 
rate gap at each maturity, $b \phi(\tau)$. To provide an intuitive illustration of the relationship between the two sensitivities, we consider various different types of distribution for $\phi(\tau)$. First, if $\phi(\tau)$ follows a uniform distribution as shown in Figure 3(a), we have $\phi(\tau)=\bar{w}=1 / T$ for all $\tau{ }^{4}$ If, for example, $T=20$ years and $\lambda=0.143$, Equation (7) implies

$$
\left\{\begin{array}{l}
\frac{\bar{b}_{L}}{b}=1 \\
\frac{\bar{b}_{S}}{b}=0.263 \\
\frac{\bar{b}_{C}}{b}=0.176 .
\end{array}\right.
$$

The values in Equation (8) suggest that the effect on output of a one percentage point change in the overnight rate is about four times as large when this change in the overnight rate is due to a change in the level gap as when it is due to a change in the slope gap.

Next, we consider the case where $\phi(\tau)$ follows the step distribution shown in Figure 3(b) and is given by

$$
\phi(\tau)= \begin{cases}w_{0}, & 0<\tau \leq T_{0} \\ w_{1}, & T_{0}<\tau \leq T_{1} \\ w_{2}, & T_{1}<\tau \leq T\end{cases}
$$

where the range $0<\tau \leq T_{0}$ represents the short term, $T_{0}<\tau \leq T_{1}$ the medium to long term, and $T_{1}<\tau \leq T$ the super-long term. If $T_{0}=2$ years, $T_{1}=10$ years, $T=20$ years, and $\lambda=0.143$, Equation (7) implies

$$
\left\{\begin{array}{l}
\frac{b_{L}}{b}=2 w_{0}+8 w_{1}+10 w_{2}=1 \\
\frac{b_{S}}{b}=1.547 w_{0}+2.513 w_{1}+1.212 w_{2} \\
\frac{b_{C}}{b}=0.356 w_{0}+1.961 w_{1}+1.206 w_{2}
\end{array}\right.
$$

Figure 4 plots the possible combinations of $w_{i}$ for $i=0,1,2$ in Equations (9) and $\bar{w}$ as six areas in a plane, with the horizontal axis representing $b_{S} / b$ and the vertical axis

\footnotetext{
${ }^{4}$ In their NYC model, Brzoza-Brzezina and Kotłowski (2014) assume a priori that $\phi(\tau)$ follows a uniform distribution. They further assume that $L_{t}^{*}$ is equal to $L_{t}$, and that $C_{t}^{*}$ is equal to the historical average of $C_{t}$.
} 
representing $b_{C} / b$. If, for example, $b_{S}>\bar{b}_{S}$ and $b_{C}=\bar{b}_{C}$, then $w_{0}>\bar{w}>w_{1}, w_{2}$, which corresponds to area (II) in the figure. This suggests that the economy is relatively sensitive to short-term yields. The slope gap can therefore be regarded as a factor that mainly affects short-term yields. If $b_{S}=\bar{b}_{S}$ and $b_{C}>\bar{b}_{C}$, then $w_{1}>\bar{w}>w_{0}, w_{2}$, which corresponds to area (VI) in the figure. This suggests that the economy is relatively sensitive to medium-term yields and indicates that the curvature gap can be regarded as a factor that mainly affects medium-term yields. The remaining gap, the level gap, can thus be regarded as a factor that mainly affects long-term yields.

$\phi(\tau)$ may follow a more complex distribution. Here, we consider the case where $\phi(\tau)$ follows a distribution consisting of two beta distributions such as

$$
\phi(\tau)=\omega f_{1}\left(\alpha_{1}, \beta_{1}\right)+(1-\omega) f_{2}\left(\alpha_{2}, \beta_{2}\right)
$$

where

$$
f_{k}\left(\alpha_{k}, \beta_{k}\right)=\frac{1}{B\left(\alpha_{k}, \beta_{k}\right)}\left(\frac{\tau}{T}\right)^{\alpha_{k}-1}\left(\frac{1-\tau}{T}\right)^{\beta_{k}-1}
$$

for $k=1,2$. $\omega$ is the weight determining the mix of the beta distributions, $f_{1}(\cdot)$ in the first term and $f_{2}(\cdot)$ in the second term of the right-hand side of Equation (10), and $B(\cdot)$ is defined as $B(\cdot) \equiv \int_{0}^{1} t^{\alpha_{k}-1}(1-t)^{\beta_{k}-1} d t$. Assuming $\alpha_{1}<1$ and $\beta_{1}, \alpha_{2}, \beta_{2}>1, f_{1}(\cdot)$ declines monotonically and $f_{2}(\cdot)$ is a unimodal distribution with a peak in the medium- to long-term zone. In this case, $\omega$ can be interpreted as the share of economic activities that are affected by short-term yields such as activities based on borrowing at floating rates. As shown in Figure 3(c) the beta mixture distribution can take a more compex form, even if the average of $\phi(\tau)$ in each range of maturities indicates a simple monotonically decreasing case with $w_{0}, w_{1}>\bar{w}>w_{2}$, which corresponds to area (I) in Figure 4.

\section{Estimation method}

To estimate the NYC model given by Equation (6), we consider the following state 
space model:

$$
\begin{aligned}
& {\left[\begin{array}{c}
y_{t} \\
L_{t} \\
S_{t} \\
C_{t}
\end{array}\right]=\left[\begin{array}{l}
y_{t}^{*} \\
L_{t}^{*} \\
S_{t}^{*} \\
C_{t}^{*}
\end{array}\right]+\left[\begin{array}{cccc}
a_{y} & b_{L} & b_{S} & b_{C} \\
0 & a_{L} & 0 & 0 \\
0 & 0 & a_{S} & 0 \\
0 & 0 & 0 & a_{C}
\end{array}\right]\left[\left(\begin{array}{c}
y_{t-1} \\
L_{t-1} \\
S_{t-1} \\
C_{t-1}
\end{array}\right)-\left(\begin{array}{c}
y_{t}^{*} \\
L_{t}^{*} \\
S_{t}^{*} \\
C_{t}^{*}
\end{array}\right)\right]+\left[\begin{array}{cccc}
1 & 0 & 0 & 0 \\
g_{y L} & 1 & 0 & 0 \\
g_{y S} & 0 & 1 & 0 \\
g_{y C} & 0 & 0 & 1
\end{array}\right]\left[\begin{array}{c}
\varepsilon_{t}^{y} \\
\varepsilon_{t}^{L} \\
\varepsilon_{t}^{S} \\
\varepsilon_{t}^{C}
\end{array}\right],} \\
& {\left[\begin{array}{c}
\Delta y_{t}^{*} \\
L_{t}^{*} \\
S_{t}^{*} \\
C_{t}^{*}
\end{array}\right]=\left[\begin{array}{l}
\Delta y_{t-1}^{*} \\
L_{t-1}^{*} \\
S_{t-1}^{*} \\
C_{t-1}^{*}
\end{array}\right]+\left[\begin{array}{cccc}
1 & 0 & 0 & 0 \\
h_{y L} & 1 & 0 & 0 \\
h_{y S} & h_{L S} & 1 & 0 \\
h_{y C} & h_{L C} & h_{S C} & 1
\end{array}\right]\left[\begin{array}{c}
\varepsilon_{t}^{\Delta y^{*}} \\
\varepsilon_{t}^{L^{*}} \\
\varepsilon_{t}^{S^{*}} \\
\varepsilon_{t}^{C^{*}}
\end{array}\right] .}
\end{aligned}
$$

The first line of Equation (11) is the IS curve, which corresponds to Equation (6) with a lagged term of the output gap, $y_{t-1}-y_{t}^{*}$, added. The second to fourth lines define the dynamics of the real yield curve. We assume that the three components of the yield curve gap, $L_{t}-L_{t}^{*}, S_{t}-S_{t}^{*}, C_{t}-C_{t}^{*}$, follow an AR(1) process. They are innovated by two types of shocks: demand shocks, $\varepsilon_{t}^{y}$, and shocks specific to the real yield curve, $\varepsilon_{t}^{L}$, $\varepsilon_{t}^{S}$, and $\varepsilon_{t}^{C}$. Note that the lagged terms of the output gap and the yield curve gap are not the difference of the observed variables at $t-1$ from the state variables at $t-1$ but from the state variables at $t$. With this setting, the real yield curve matches the natural yield curve in the stationary state, i.e., $L_{t}-L_{t}^{*}=S_{t}-S_{t}^{*}=C_{t}-C_{t}^{*}=0$, and the actual output will converge to the potential output, i.e., $y_{t}=y_{t}^{*}$.

Equation (12) is the state equation, which describes the dynamics of potential growth, $\Delta y_{t}^{*}$, and the components of the natural yield curve, $L_{t}^{*}, S_{t}^{*}$, and $C_{t}^{*}$. This equation means that the level and shape of the natural yield curve vary depending on economic activity and expectations of future economic growth. The shock, $\varepsilon_{t}^{\Delta y^{*}}$, is the innovation to potential growth, which also simultaneously affects the natural yield curve. A positive $\varepsilon_{t}^{\Delta y^{*}}$ increases $\Delta y_{t}^{*}$ and then $y_{t}^{*}$, and raises the level of the natural yield curve, and vice versa. The remaining three shocks, $\varepsilon_{t}^{L^{*}}, \varepsilon_{t}^{S^{*}}$, and $\varepsilon_{t}^{C^{*}}$, include all the other elements which affect the natural yield curve, such as changes in expectations of future economic growth. For example, heightened uncertainty regarding future economic conditions will lower the natural yield curve through a negative $\varepsilon_{t}^{L^{*}}$. Moreover, if future economic growth is expected to be higher than current growth, the 
natural yield curve will be steeper through a positive $\varepsilon_{t}^{L^{*}}$ and a negative $\varepsilon_{t}^{S^{*}}$ or $\varepsilon_{t}^{C^{*}}$. Conversely, if future economic growth is expected to be lower, the natural yield curve will be flatter through a negative $\varepsilon_{t}^{L^{*}}$ and a positive $\varepsilon_{t}^{S^{*}}$ or $\varepsilon_{t}^{C^{*}}$. We identify these three shocks by assuming the lower triangular matrix, which specifies the simultaneous relations among the shocks, in the second term of the right-hand side of Equation (12). ${ }^{5}$

Following Brzoza-Brzezina and Kotłowski (2014), we estimate the natural yield curve in two steps. In the first step, we use the dynamic Nelson-Siegel (DNS) model (Diebold and Li, 2006) to decompose $\left\{r_{\tau, t}\right\}_{\tau=0}^{T}$ into $L_{t}, S_{t}$, and $C_{t}$. Specifically, we estimate the following state space model:

$$
\begin{aligned}
& r_{\tau, t}=L_{t}+S_{t} \frac{1-e^{-\lambda \tau}}{\lambda \tau}+C_{t}\left(\frac{1-e^{-\lambda \tau}}{\lambda \tau}-e^{-\lambda \tau}\right)+\varepsilon_{\tau, t}, \\
& {\left[\begin{array}{lll}
L_{t}- & \mu_{L} \\
S_{t}- & \mu_{S} \\
C_{t}- & \mu_{C}
\end{array}\right]=\left[\begin{array}{lll}
a_{11} & a_{12} & a_{13} \\
a_{21} & a_{22} & a_{23} \\
a_{31} & a_{32} & a_{33}
\end{array}\right]\left[\begin{array}{lll}
L_{t-1} & -\mu_{L} \\
S_{t-1} & - & \mu_{S} \\
C_{t-1} & - & \mu_{C}
\end{array}\right]+\left[\begin{array}{l}
\xi_{t}^{L} \\
\xi_{t}^{S} \\
\xi_{t}^{C}
\end{array}\right],}
\end{aligned}
$$

where $L_{t}, S_{t}$, and $C_{t}$ follow a $\operatorname{VAR}(1)$ process with means of $\mu_{L}, \mu_{S}$, and $\mu_{C}$, respectively. We assume that both $\varepsilon_{\tau, t}$ and $\xi_{t}^{j}$ for $j=L, S, C$ follow a normal distribution. Following Diebold et al. (2006), we assume that the $\xi_{t}^{j}$ are mutually correlated, while the $\varepsilon_{\tau, t}$ are mutually independent and uncorrelated with $\xi_{t}^{j}$. We estimate $L_{t}, S_{t}$, and $C_{t}$ based on the observation equation (13) and the state equation (14) using the Kalman filter method.

In the second step, we estimate the NYC model. Following Clark and Kozicki (2005), who estimate the natural rate of interest, we treat $y_{t}^{*}$ and $\Delta y_{t}^{*}$ as observed variables. Given $y_{t}^{*}$ and $\Delta y_{t}^{*}$ as well as $L_{t}, S_{t}$, and $C_{t}$ estimated from the DNS model in the first step, Equations (11) and (12) are rewritten in the following form:

\footnotetext{
${ }^{5}$ We arrange $L_{t}^{*}, S_{t}^{*}$, and $C_{t}^{*}$ in descending order of the size of their impact on the natural yield curve. Alternatively, one could assume that the natural yield curve is determined from long to short maturities and arrange $L_{t}^{*}, C_{t}^{*}$, and $S_{t}^{*}$ in the corresponding order. We also estimated the model using the latter ordering and found that the estimation results remained essentially unchanged.
} 


$$
\begin{aligned}
& {\left[\begin{array}{c}
y_{t}-y_{t}^{*} \\
L_{t} \\
S_{t} \\
C_{t}
\end{array}\right]=\left[\begin{array}{cccc}
a_{y} & b_{L} & b_{S} & b_{C} \\
0 & a_{L} & 0 & 0 \\
0 & 0 & a_{S} & 0 \\
0 & 0 & 0 & a_{C}
\end{array}\right]\left[\begin{array}{c}
y_{t-1}-y_{t}^{*} \\
L_{t-1} \\
S_{t-1} \\
C_{t-1}
\end{array}\right]} \\
& +\left[\begin{array}{ccc}
b_{L} & b_{S} & b_{C} \\
1-a_{L} & 0 & 0 \\
0 & 1-a_{S} & 0 \\
0 & 0 & 1-a_{C}
\end{array}\right]\left[\begin{array}{c}
L_{t}^{*} \\
S_{t}^{*} \\
C_{t}^{*}
\end{array}\right]+\left[\begin{array}{cccc}
1 & 0 & 0 & 0 \\
g_{y L} & 1 & 0 & 0 \\
g_{y S} & 0 & 1 & 0 \\
g_{y C} & 0 & 0 & 1
\end{array}\right]\left[\begin{array}{c}
\varepsilon_{t}^{y} \\
\varepsilon_{t}^{L} \\
\varepsilon_{t}^{S} \\
\varepsilon_{t}^{C}
\end{array}\right] \\
& {\left[\begin{array}{l}
L_{t}^{*} \\
S_{t}^{*} \\
C_{t}^{*}
\end{array}\right]=\left[\begin{array}{l}
L_{t-1}^{*} \\
S_{t-1}^{*} \\
C_{t-1}^{*}
\end{array}\right]+\left[\begin{array}{l}
h_{y L} \\
h_{y S} \\
h_{y C}
\end{array}\right] \varepsilon_{t}^{\Delta y^{*}}+\left[\begin{array}{ccc}
1 & 0 & 0 \\
h_{L S} & 1 & 0 \\
h_{L C} & h_{S C} & 1
\end{array}\right]\left[\begin{array}{c}
\varepsilon_{t}^{L^{*}} \\
\varepsilon_{t}^{S^{*}} \\
\varepsilon_{t}^{C^{*}}
\end{array}\right]}
\end{aligned}
$$

Because $\Delta y_{t}^{*}$ represents actually observed values, so does its lagged difference, $\varepsilon_{t}^{\Delta y^{*}} \equiv \Delta y_{t}^{*}-\Delta y_{t-1}^{*}$. We assume that each of the remaining seven shocks, $\varepsilon_{t}^{y}, \varepsilon_{t}^{L}, \varepsilon_{t}^{S}$, $\varepsilon_{t}^{C}, \varepsilon_{t}^{L^{*}}, \varepsilon_{t}^{S^{*}}$, and $\varepsilon_{t}^{C^{*}}$, follows a normal distribution and that they are mutually independent. We estimate $L_{t}^{*}, S_{t}^{*}$, and $C_{t}^{*}$ based on the observation equation (15) and the state equation (16) using the Kalman filter method.

\section{Empirical analysis}

\subsection{Data}

This section applies the DNS and NYC models developed in the previous section to data for Japan. The data used for estimating the models are quarterly series of real zero-coupon rates, the output gap, and the potential growth rate from 1992/Q3 to 2014/Q4. Developments in the series during our observation period are shown in Figure 5. Specifically, for the real zero-coupon rate we use the nominal zero-coupon rate deflated by inflation expectations for each maturity obtained from the Consensus Forecasts reported by Consensus Economics Inc. ${ }^{6}$ Because inflation expectations are

\footnotetext{
${ }^{6}$ Due to data limitations, we use nominal interest rates deflated by survey-based inflation expectations instead of directly observed market-based real interest rates. The estimates reported below, in particular the estimated levels of the natural yield curve and the actual real yield curve,
} 
surveyed only for a period of up to 10 years ahead, we assume that inflation expectations for more than 10 years ahead are the same as those for 6-10 years ahead. Moreover, we linearly interpolate the biannual series of inflation expectations to obtain the quarterly series. As the terms to maturity for real zero-coupon rates, we select 1, 2, 3, 7, 10, and 20 years. The output gap and potential growth series are estimated by the Bank of Japan.

\subsection{Estimated model parameters}

Table 1(a) reports the model parameters estimated from the DNS model. The means of the level and slope factors are $\mu_{L}=0.019$ and $\mu_{S}=-0.009$, respectively. This suggests that the average of the real yield curve over the past 20 years is a moderately upward sloping curve with a real yield of 1.9 percent at the long end and 0.9 percent at the short end. ${ }^{7}$ For the mean of the curvature factor, we obtain $\mu_{C}<0$, which implies that the historical average of the real yield curve bends downward.

Table 1(b) shows the model parameters estimated from the NYC model. The parameters associated with the yield curve gap, $b_{L}, b_{S}$, and $b_{C}$, are all negative, thus satisfying the sign restrictions, and statistically significant. Based on the estimates of $b_{L}$, $b_{S}, b_{C}$, and $\lambda$, Equations (8) and (9) imply the following relationships:

$$
\begin{aligned}
& b_{S} / b=0.543>\bar{b}_{S} / b=0.263, \text { and } \\
& b_{C} / b=0.209>\bar{b}_{C} / b=0.176,
\end{aligned}
$$

which implies $w_{0}, w_{1}>\bar{w}>w_{2}{ }^{8}$. Note that this case corresponds to area (I) in Figure

should therefore be interpreted with some latitude. For example, the 10-year real interest rate of Japanese government inflation-indexed bonds (JGBi) was -0.5 percent at the end of 2014, while the corresponding rate plotted in Figure 5 is -1.1 percent. In addition, adjustments for the direct effects of the consumption tax hikes on inflation expectations are made prior to the analysis. Specifically, we extrapolate 1-year inflation expectations based on a spline curve. The curve is fitted to inflation expectations for 2 and more years ahead, which are not affected by the consumption tax hikes.

${ }^{7}$ From $\lim _{\tau \rightarrow+\infty} r_{\tau, t}=L_{t}$ and $\lim _{\tau \rightarrow 0} r_{\tau, t}=L_{t}+S_{t}, \mu_{L}$ and $\mu_{L}+\mu_{S}$ correspond to the historical average of the long- and short-term interest rates, respectively.

${ }^{8}$ In this section we focus on the relative size of $\phi(\tau)$ for each $\tau$. Although, more strictly speaking, the lagged effect of the output gap should also be taken into account when discussing $\phi(\tau)$, we omit it here. 
4. The estimated $b_{L}, b_{S}$, and $b_{C}$ suggest that $\phi(\tau)$ is a downward sloping distribution as a whole, and that for maturities of up to 10 years the sensitivity of the economy to the interest rate gap is higher than in the case of $\phi(\tau)=\bar{w}$ and lower for maturities of more than 10 years.

In Japan, the ratio of floating rate loans to total loans made by major banks stands at over 50 percent. For those floating rate loans the main reference rate is the Tokyo Interbank Offered Rate (Tibor), so that most economic activities are likely to be affected by short-term interest rates. Against this background, we assume in Equation (10) that $\omega=0.5$ and that the value of $\phi(\tau)$ at $\tau=1$ year is sufficiently larger than the values at other maturities. In this case, as shown in Figure $3(\mathrm{c})$, the beta distribution $f_{1}(\cdot)$ becomes a monotonically decreasing distribution with a peak at $\tau=1$ year, while $f_{2}(\cdot)$ has a peak at $\tau=5$ years. ${ }^{9}$ These shapes of the estimated $f_{1}(\cdot)$ and $f_{2}(\cdot)$ have the following implications. Those borrowing at floating rates are particularly sensitive to the interest rate gap for a horizon of one year. On the other hand, those borrowing at fixed rates are relatively sensitive to the interest rate gap for a horizon of 3 to 5 years, which corresponds to the average length of the business cycle. Both groups are less sensitive to the interest rate gap for a longer horizon as their sensitivity to the interest rate gap decreases monotonically as the length of maturity increases.

\subsection{Developments in yield curves}

Figure 6 plots developments in the estimated components of the natural yield curve and the actual real yield curve. For both curves, the level factor has been declining modestly since the 1990s and the slope factor has been increasing. The 90 percent confidence intervals imply that each of these factors of the natural yield curve has been zero since 2012. Meanwhile, the curvature factor of the natural yield curve has been slightly negative but stable, while that of the actual real yield curve has been volatile in negative territory.

\footnotetext{
${ }^{9}$ The mixture distribution depicted in Figure 3(c) is the simple average of the distributions $f_{1}\left(\alpha_{1}, \beta_{1}\right)$ and $f_{2}\left(\alpha_{2}, \beta_{2}\right)$ for $0<\alpha_{1}<1$ and $1<\beta_{1} \leq 10$, which are consistent with the estimated $b_{S} / b$ and $b_{C} / b$ under the conditions that $\omega=0.5$ and $\phi(\tau=1$ year $)-\phi(\tau>$ 1 year) $>0.1$.
} 
The combination of $L_{t}^{*}, S_{t}^{*}$, and $C_{t}^{*}$ yields the natural rate of interest at any maturity, i.e., the natural yield curve, which is displayed in Figure 7. The estimated natural yield curve has two notable features. The first is that the level of the natural yield curve has been shifting downward. In line with the decline in the level factor, the level of the natural yield curve, which was about two percent in the middle of the 1990s, has been around zero percent since the global financial crisis. In fact, the natural yields for maturities of less than 10 years fell into negative territory in 2012. The second notable feature is the flattening of the natural yield curve. Due to the decline in the negative slope factor, the term spread between long- and short-term natural yields, which was about 150 basis points at its historical high, was almost zero in 2014. Both the downward shift and the flattening of the natural yield curve are attributable to the downward revision of market views on current economic activity and the future outlook.

\subsection{Comparison of monetary easing programs}

Since the late 1990s, four monetary easing programs have been implemented in Japan: the zero interest rate policy from February 1999 to August 2000, the quantitative easing policy from March 2001 to March 2006, the comprehensive monetary easing policy from October 2010 to April 2013, and the quantitative and qualitative monetary easing (QQE) policy from April 2013 onward. In each program, the Bank of Japan chose appropriate policy measures from various alternatives: setting the policy rate at effectively zero, changing the main operating target to the current account balance at the Bank of Japan and the monetary base, and purchasing long-term government bonds and risky assets.

Figure 8 shows the relationship between the natural yield curve and the actual real yield curve for each of these four programs. During the zero interest rate policy period, the Bank of Japan cut its policy rate to effectively zero through the short-term interest rate control and induced a negative yield curve gap mainly for short maturities. The next two programs, the quantitative easing and the comprehensive monetary easing programs, resulted in a negative gap for medium rather than short maturities. This implies that the monetary easing effects of these two programs were achieved mainly through the Bank 
of Japan's commitment to keep its policy expansionary for some time. ${ }^{10}$ In addition, in the case of the comprehensive monetary easing program, the Bank of Japan's purchases of government bonds with maturities of up to 3 years also contributed to the decline in yields for medium maturities.

Finally, the current QQE program, which involves large-scale purchases of long-term government bonds by the Bank of Japan, has resulted in a considerable negative yield curve gap for long and super-long maturities as well as short and medium maturities. Consequently, the QQE program has led to the most accommodative financial conditions of the four programs. Specifically, as shown in Figure 9, the actual 10-year real yield has declined considerably in the nearly two years since the introduction of the program, while the corresponding natural yield has been flat, causing the interest rate gap between the two to expand by about 90 basis points from 20 basis points to 110 basis points. This is the fastest pace of expansion during any of the four programs.

\subsection{Measuring the interest rate environment}

To measure how accommodative financial conditions are, we construct an indicator of the interest rate environment (IIE), which aggregates information on the interest rate gaps for all maturities. We define the IIE as the potential effects on the current and future output gap of the yield curve gap at each point in time. Specifically, the IIE, $I_{t}$, is defined by the sum of the contributions of the shocks specific to the real yield curve, $\varepsilon_{t}^{L}$, $\varepsilon_{t}^{S}$, and $\varepsilon_{t}^{C}$. That is,

$$
\begin{aligned}
I_{t} & \equiv \sum_{i=0}^{\infty} a_{y}^{i} \cdot\left[b_{L} \varepsilon_{t}^{L} \sum_{i=0}^{\infty} a_{L}^{i}+b_{S} \varepsilon_{t}^{S} \sum_{i=0}^{\infty} a_{S}^{i}+b_{C} \varepsilon_{t}^{C} \sum_{i=0}^{\infty} a_{C}^{i}\right] \\
& =\frac{1}{1-a_{y}}\left(\frac{b_{L}}{1-a_{L}} \varepsilon_{t}^{L}+\frac{b_{S}}{1-a_{S}} \varepsilon_{t}^{S}+\frac{b_{C}}{1-a_{C}} \varepsilon_{t}^{C}\right) .
\end{aligned}
$$

Note that $\varepsilon_{t}^{L}, \varepsilon_{t}^{S}$, and $\varepsilon_{t}^{C}$ reflect monetary policy shocks. If $I_{t}=0$, the monetary policy stance can be interpreted as neutral. On the other hand, if $I_{t}>0$, the monetary

\footnotetext{
${ }^{10}$ For the quantitative easing program, the policy commitment was to maintain the program until the consumer price index registers stably at zero percent or shows an increase year on year, while for the comprehensive monetary easing program it was to maintain the program until the Bank of Japan judges that the 1 percent goal is in sight.
} 
policy stance can be viewed as accommodative and as conducive to improving the output gap. Conversely, if $I_{t}<0$, the monetary policy stance can be regarded as contractionary. Unlike interest rate gaps for a single maturity, the IIE derived from the entire yield curve provides a good indicator of developments in financial conditions overall. As shown in Figure 10, developments in the IIE are similar to those in firms' funding conditions, another indicator measuring financial conditions overall reported in the Tankan by the Bank of Japan.

Decomposition of the IIE indicates that during the zero interest rate policy period the steepening of the yield curve due to the zero policy rate was the most effective in improving the output gap, while the bending of the yield curve due to the Bank of Japan's commitment policy aiming to influence medium-term yields was the most effective during the quantitative easing and the comprehensive monetary easing programs. $^{11}$ On the other hand, under the QQE program, the Bank of Japan's long-term government bond purchases have played a key role in the downward shift of the yield curve, and thereby improved the output gap. Our natural yield curve model implies that the impact of the overall QQE program on the output gap, measured by an increase in $I_{t}$, is equivalent to the possible impact of a steepening of the yield curve with a reduction in the short-term interest rate of 190 basis points.

\section{Concluding remarks}

This paper illustrated the concept of the natural yield curve and an approach to measuring it. Our NYC model allows us to estimate the natural yields for all maturities using information on the entire yield curve and distinguish the following differences: the difference between the sensitivity of the economy to various shapes of the yield curve gap, and the difference in the term structure between the natural yield curve and the actual real yield curve. The yield curve gap can be used to measure the financial conditions implied not only by interest rates at a specific maturity but also by the entire

${ }^{11}$ In Figure 10, the contributions of $\varepsilon_{t}^{L}, \varepsilon_{t}^{S}$, and $\varepsilon_{t}^{C}$ to $I_{t}$ are referred to as a downward shift, a steepening, and a bending of the yield curve, respectively. 
yield curve.

However, existing monetary policy models such as dynamic stochastic general equilibrium (DSGE) models as well as monetary policy rules such as the Taylor rule do not take the term structure of natural yields into account. This means that we cannot apply those existing models and rules to the natural yield curve, which contains information on interest rates for all maturities. From both a theoretical and practical viewpoint, it is important to extend and generalize those existing models and rules to incorporate the natural yield curve. Such an extension remains a task for future work. 


\section{References}

Barsky, R., A. Justiniano, and L. Melosi (2014), "The natural rate of interest and its usefulness for monetary policy," American Economic Review: Papers \& Proceedings, 104(5), pp. 37-43.

Bomfim, A. N. (1997), "The equilibrium Fed funds rate and the indicator properties of term-structure spreads," Economic Inquiry, 35(4), pp. 830-846.

Brzoza-Brzezina, M. (2003), "Estimating the natural rate of interest: A SVAR approach," Working Paper 27, National Bank of Poland.

Brzoza-Brzezina, M. and J. Kotłowski (2014), "Measuring the natural yield curve," Applied Economics, 46(17), pp. 2052-2065.

Clark, T. E. and S. Kozicki (2005), "Estimating equilibrium real interest rates in real time," North American Journal of Economics and Finance, 16(3), pp. 395-413.

Cuaresma, J., E. Gnan, and D. Ritzberger-Gruenwald (2004), "Searching for the natural rate of interest: A euro area perspective," Empirica, 31(2-3), pp. 185-204.

Diebold, F. X. and C. Li (2006), "Forecasting the term structure of government bond yields," Journal of Econometrics, 130(2), pp. 337-364.

Diebold, F. X., G. D. Rudebusch, and S. B. Aruoba (2006), "The macroeconomy and the yield curve: A dynamic latent factor approach," Journal of Econometrics, 131(1), pp. 309-338.

Justiniano, A. and G. E. Primiceri (2010), "Measuring the equilibrium real interest rate," Economic Perspectives, 34(1), pp. 14-27.

Kamada, K. (2009), "Japan's equilibrium real interest rate," in K. Fukao (ed.), Macroeconomy and Industrial Structures, pp. 387-427, Keio University Press (in Japanese).

King, M. and D. Low (2014), "Measuring the 'world' real interest rate," NBER Working Paper 19887. 
Laubach, T. and J. C. Williams (2003), "Measuring the natural rate of interest," Review of Economics and Statistics, 85(4), pp. 1063-1070.

Neiss, K. S. and E. Nelson (2003), "The real-interest-rate gap as an inflation indicator," Macroeconomic Dynamics, 7(2), pp. 239-262.

Nelson, C. R. and A. F. Siegel (1987), "Parsimonious modeling of yield curves," Journal of Business, 60(4), pp. 473-489.

Oda, N. and J. Muranaga (2003), "On the natural rate of interest: Theory and estimates," Bank of Japan Working Paper Series, No. 03-J-5 (in Japanese). 
(a) DNS model

\begin{tabular}{ccc}
\hline Parameter & Estimate (std. err.) \\
\hline$a_{11}$ & $0.857(0.033)$ \\
$a_{21}$ & $0.150(0.052)$ \\
$a_{31}$ & $0.061(0.105)$ \\
$a_{12}$ & $0.005(0.033)$ \\
$a_{22}$ & $0.927(0.051)$ \\
$a_{32}$ & $0.124(0.106)$ \\
$a_{13}$ & $-0.081(0.014)$ \\
$a_{23}$ & $0.099(0.022)$ \\
$a_{33}$ & $0.985(0.043)$ \\
$\mu_{L}$ & $0.019(0.001)$ \\
$\mu_{S}$ & $-0.009(0.001)$ \\
$\mu_{C}$ & $-0.038(0.002)$ \\
$\lambda$ & $0.143(0.001)$ \\
\hline
\end{tabular}

(b) NYC model

\begin{tabular}{|c|c|}
\hline Parameter & Estimate (std. err.) \\
\hline$b_{L}$ & $-0.215(0.051)$ \\
\hline$b_{S}$ & $-0.117(0.014)$ \\
\hline$b_{C}$ & $-0.045(0.018)$ \\
\hline$a_{y}$ & $0.924(0.032)$ \\
\hline$a_{L}$ & $0.847(0.090)$ \\
\hline$a_{S}$ & $0.844(0.053)$ \\
\hline$a_{C}$ & $0.893(0.025)$ \\
\hline$g_{y L}$ & 0.061 (0.017) \\
\hline$g_{y s}$ & $0.064(0.019)$ \\
\hline$g_{y c}$ & $0.059(0.244)$ \\
\hline$h_{y L}$ & $0.921(0.055)$ \\
\hline$h_{y S}$ & $-0.318(0.241)$ \\
\hline$h_{y c}$ & $-0.032(0.069)$ \\
\hline$h_{L S}$ & $-0.291(0.253)$ \\
\hline$h_{L C}$ & $-0.906(0.705)$ \\
\hline$h_{S C}$ & $-1.371(1.377)$ \\
\hline
\end{tabular}

Table 1. Estimates of the model parameters 


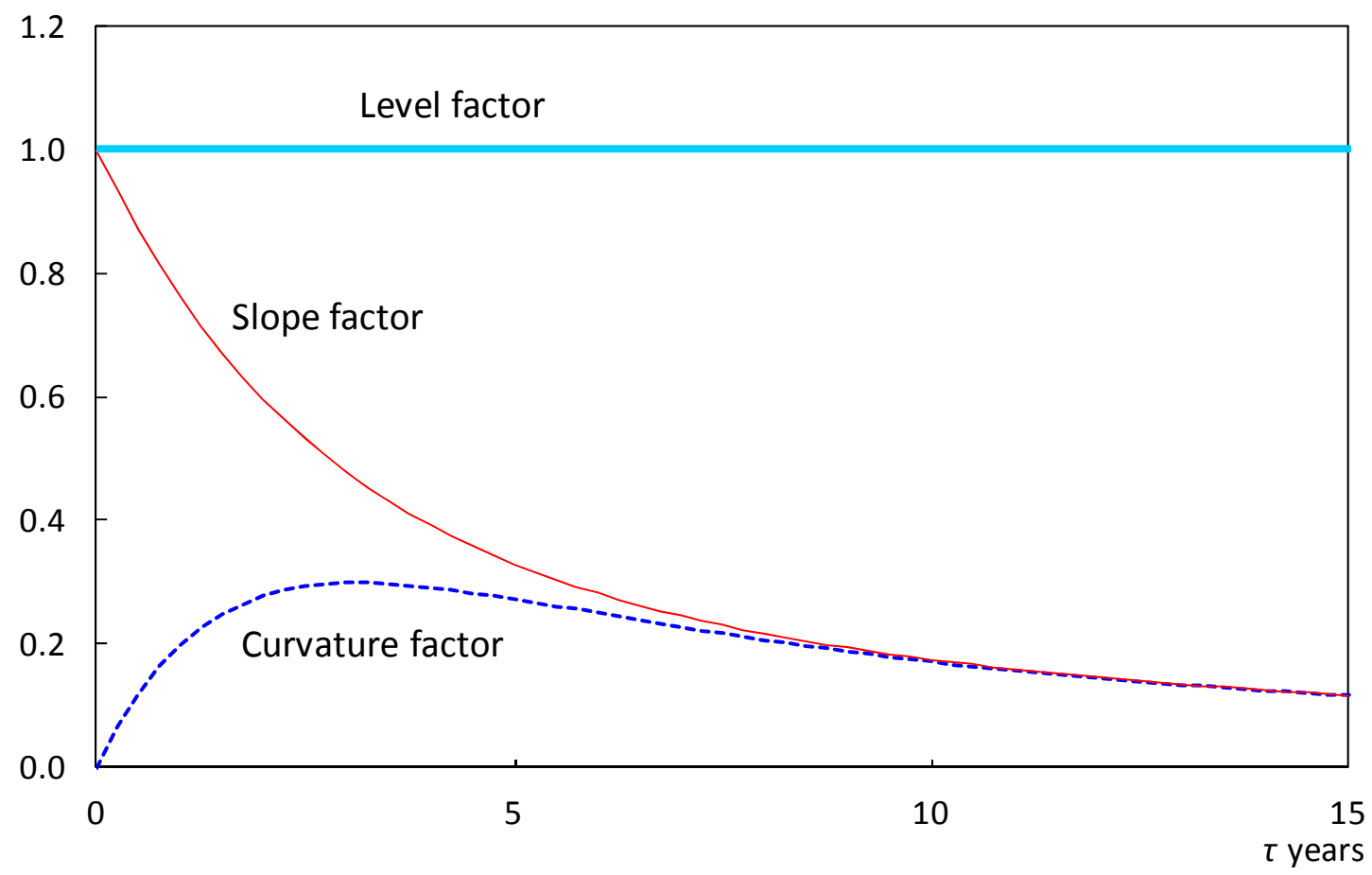

Figure 1. Factor loadings in the Nelson-Siegel model

The figure plots the case of $\lambda=0.143$. 

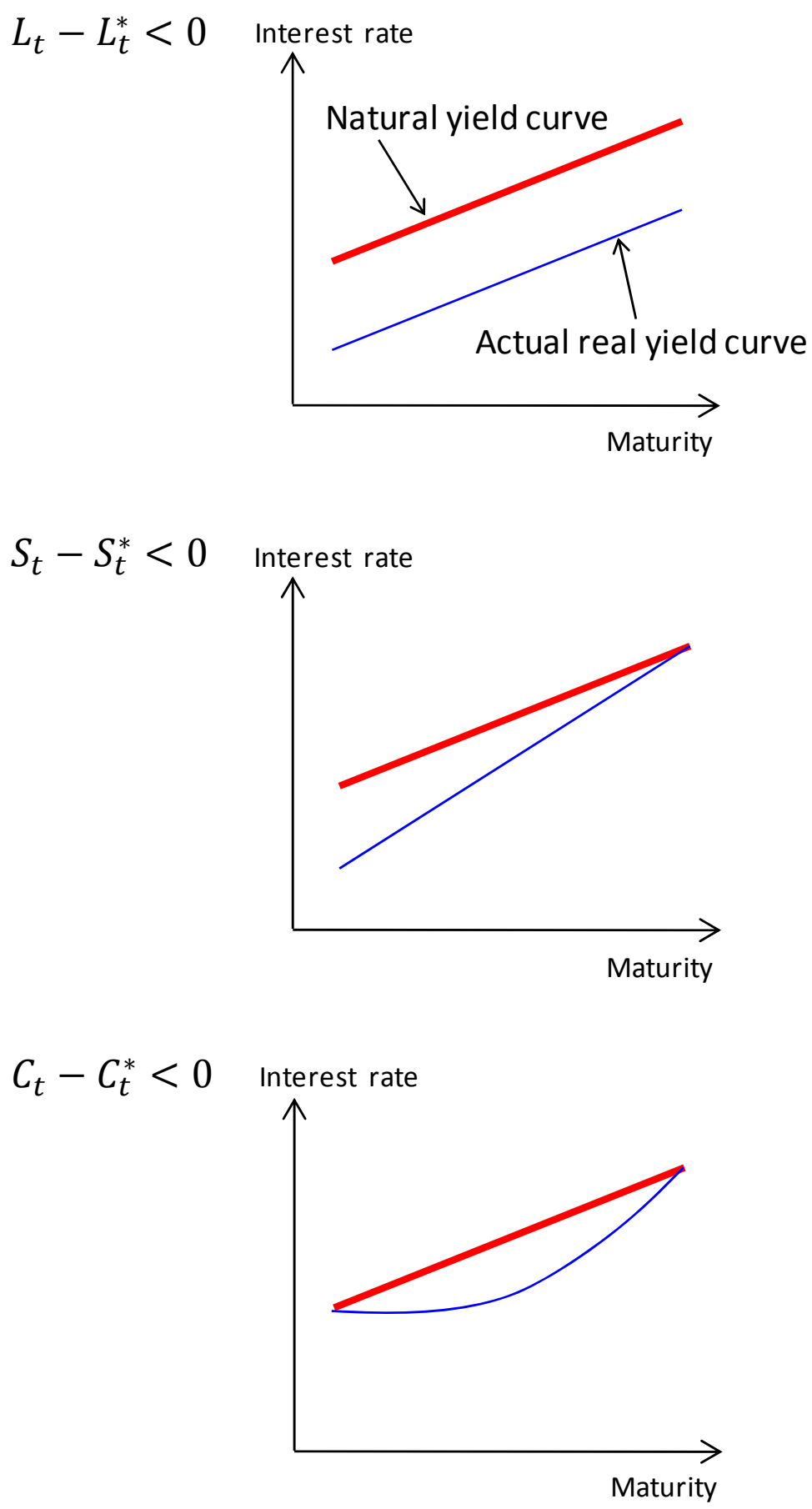

Figure 2. Illustration of the yield curve gap 
(a) Uniform distribution

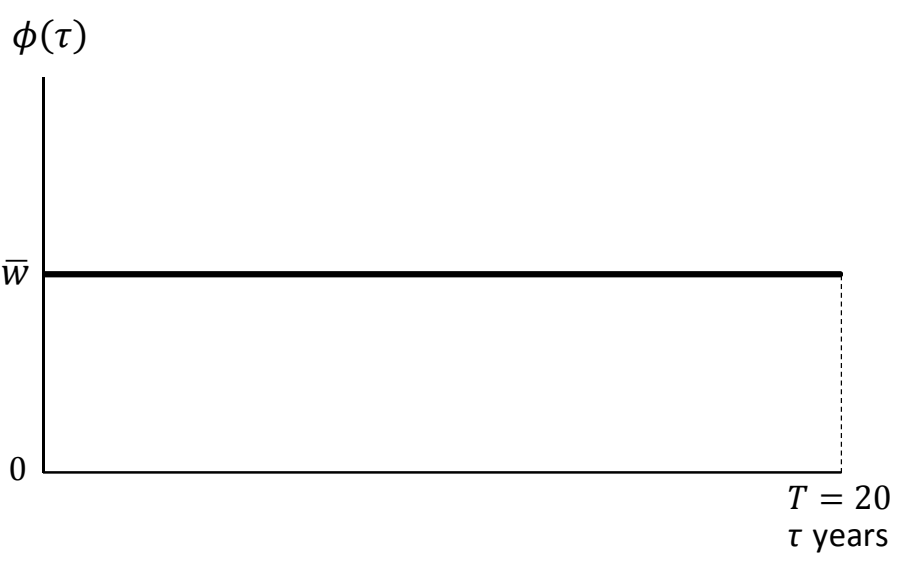

(b) Step distribution

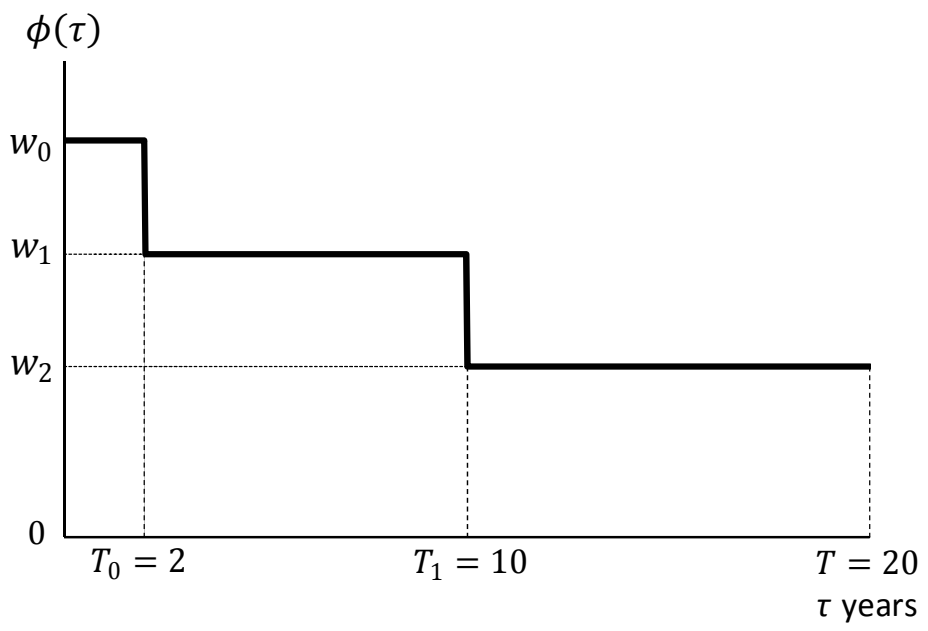

(c) Beta mixture distribution

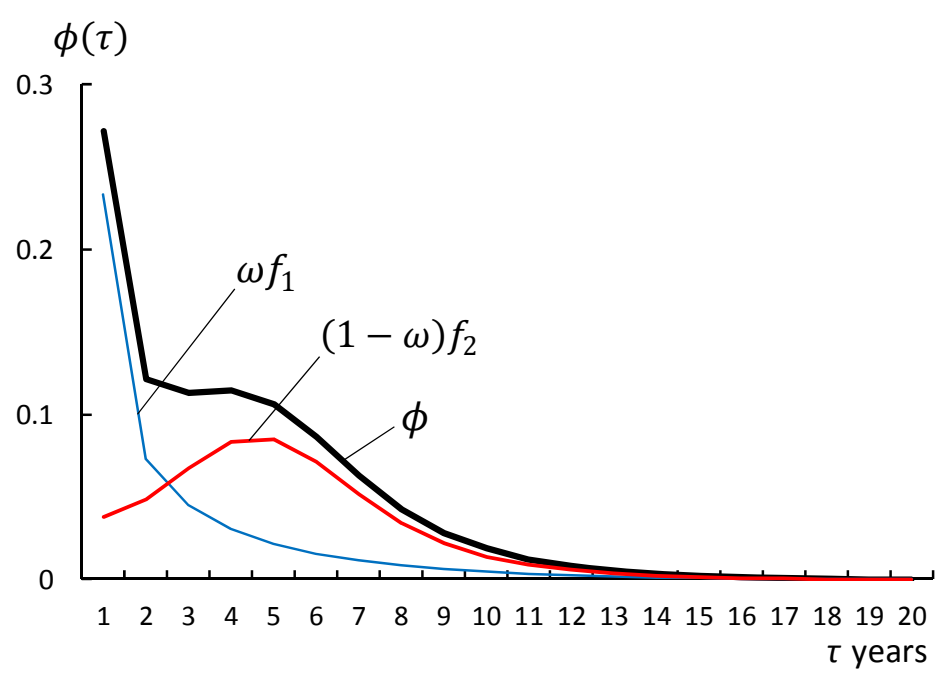

Figure 3. Distribution of $\phi(\tau)$ 


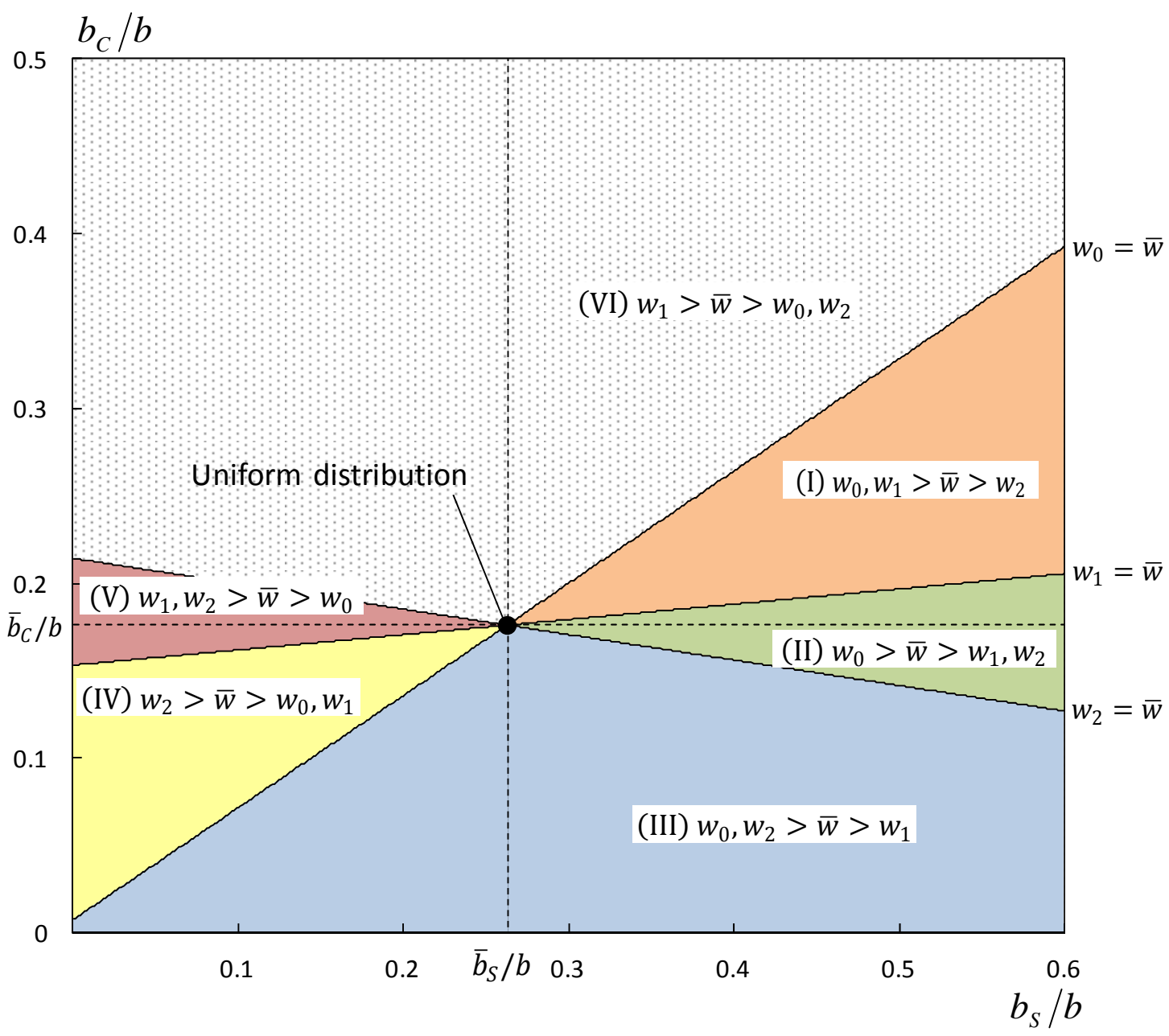

Figure 4. Domain of $w_{i}$

The figure plots the case of $T_{0}=2$ years, $T_{1}=10$ years, $T_{2}=20$ years, and $\lambda=0.143$. 
(a) Real zero coupon rates

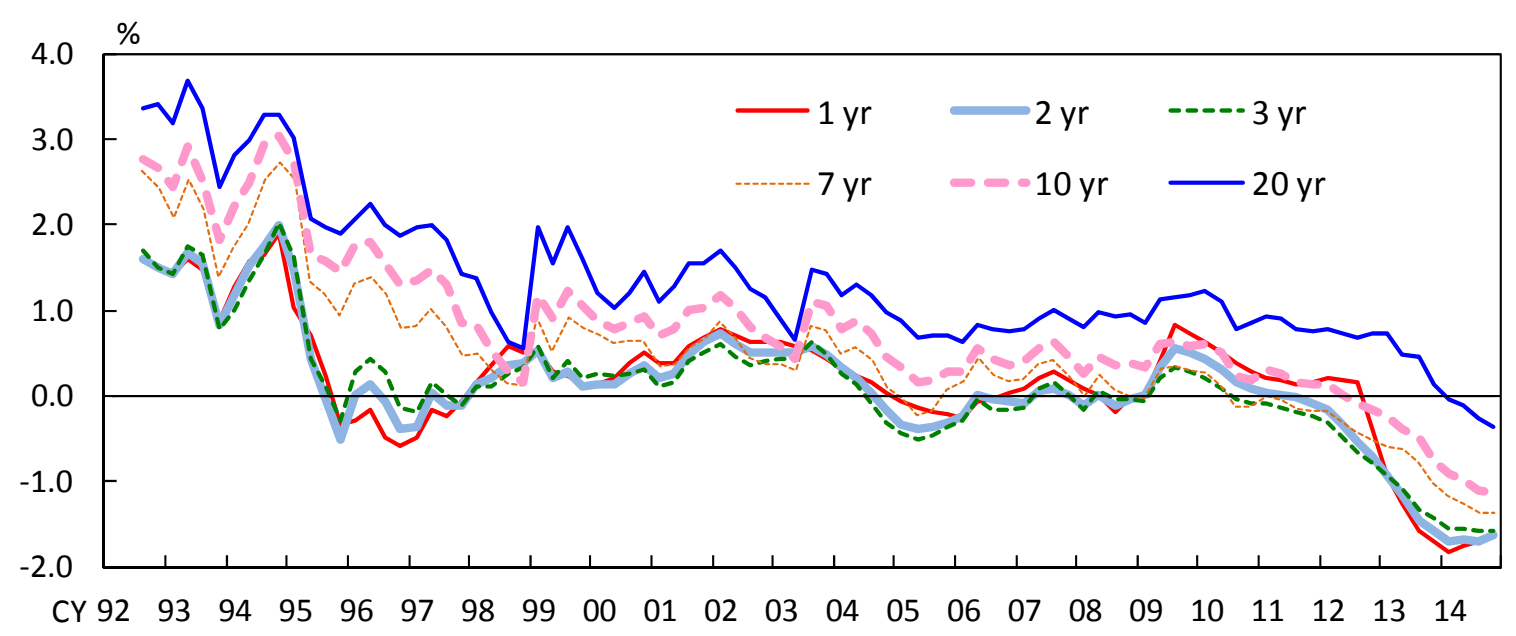

(b) Output gap

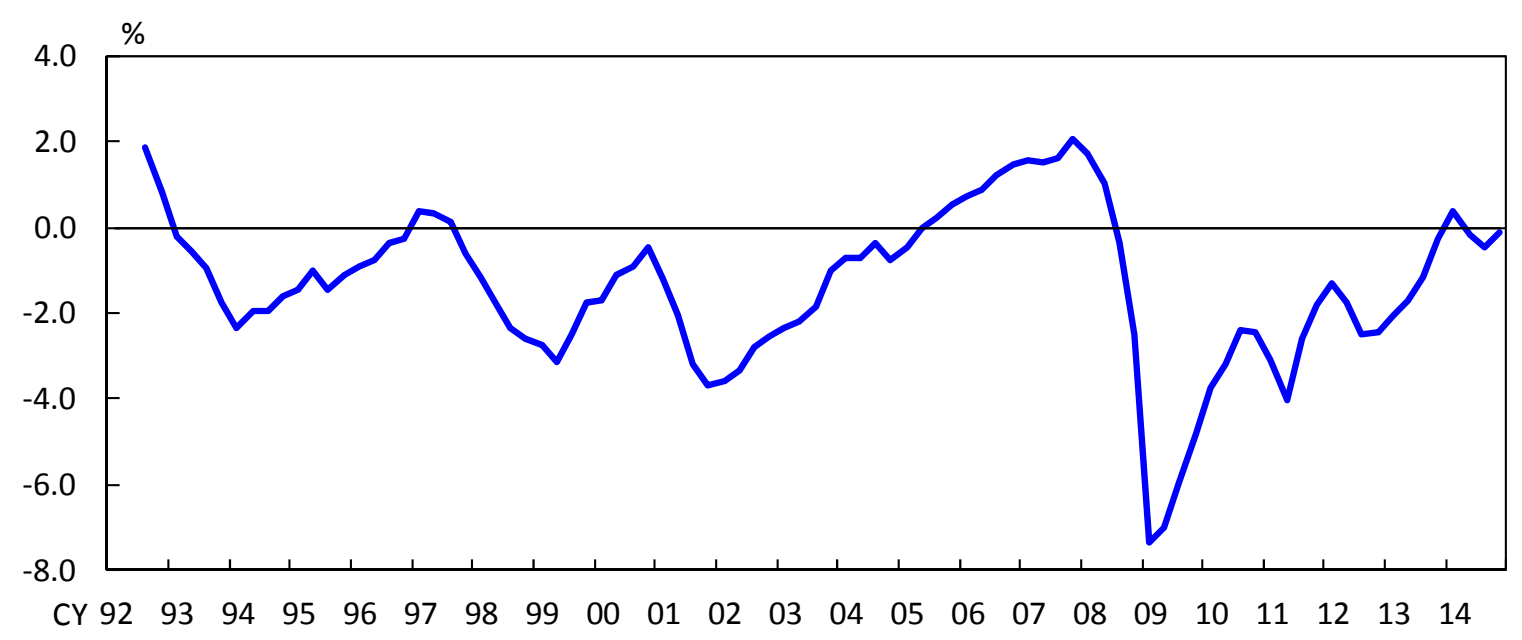

(c) Potential growth rate

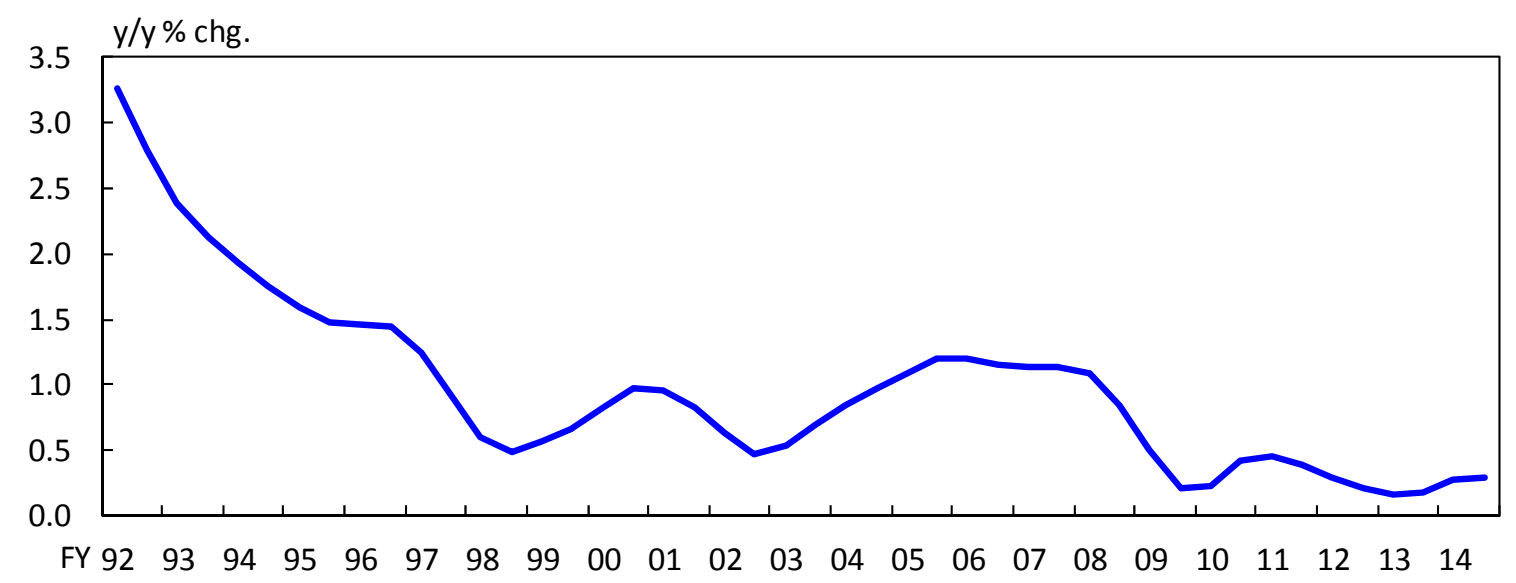

Figure 5. Data used for estimation 
(a) Level factor

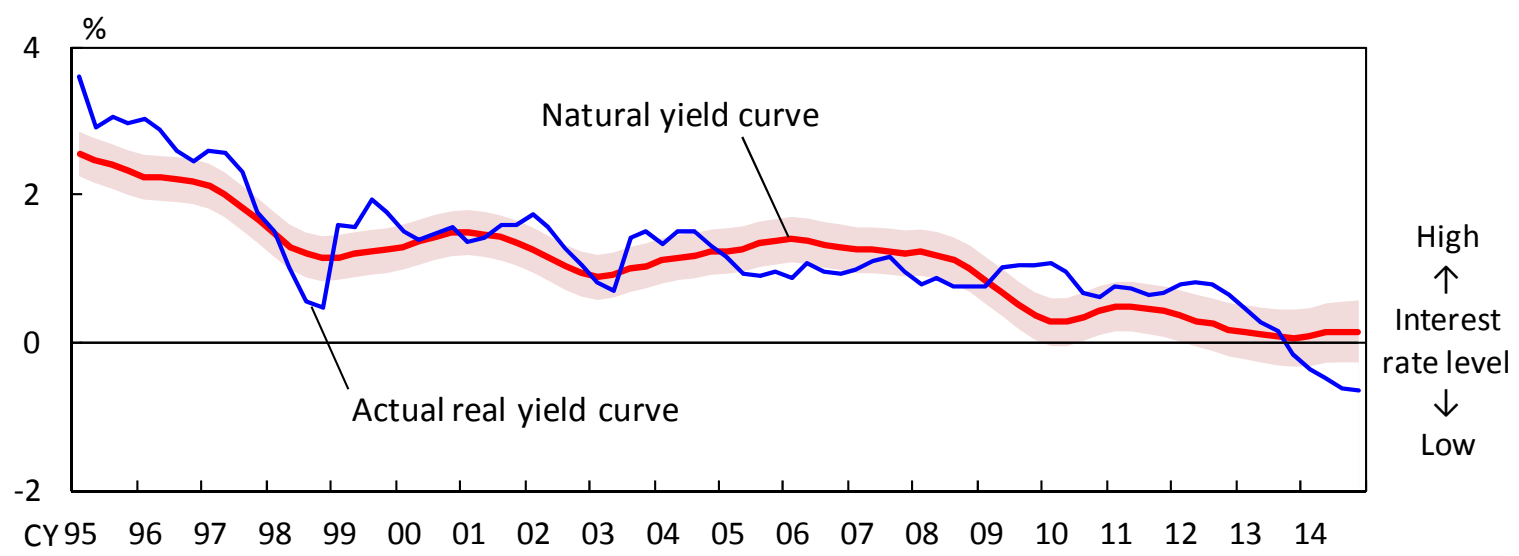

(b) Slope factor

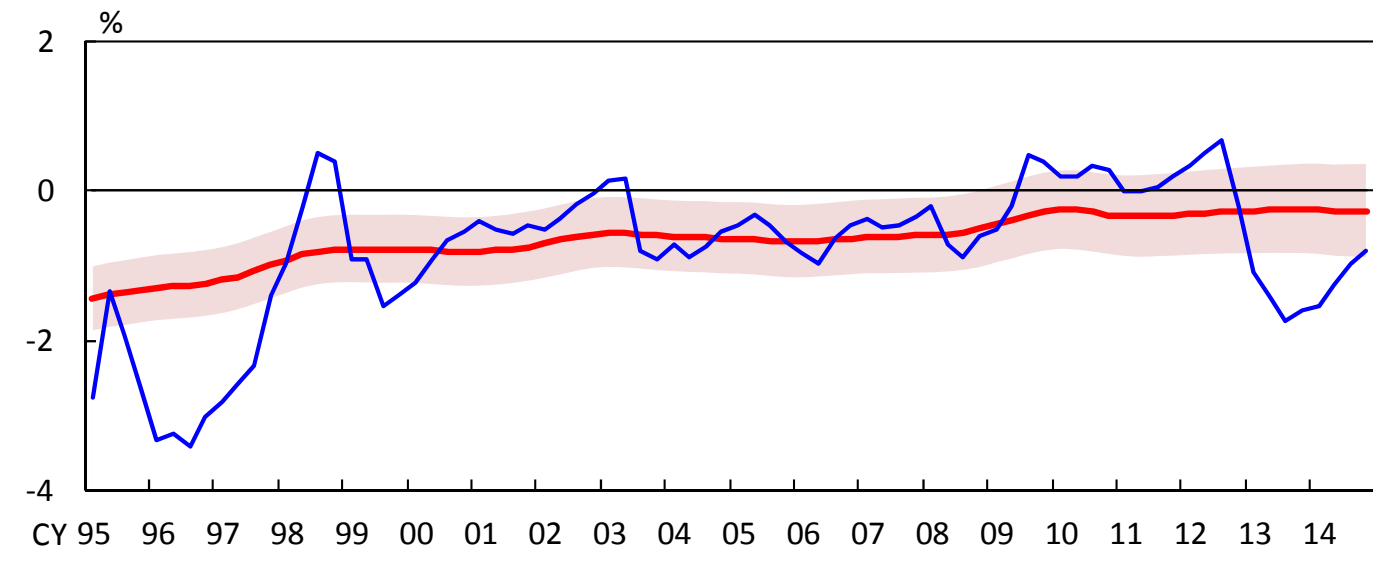

Downwardsloping $\uparrow$ $\downarrow$ Upwardsloping

(c) Curvature factor

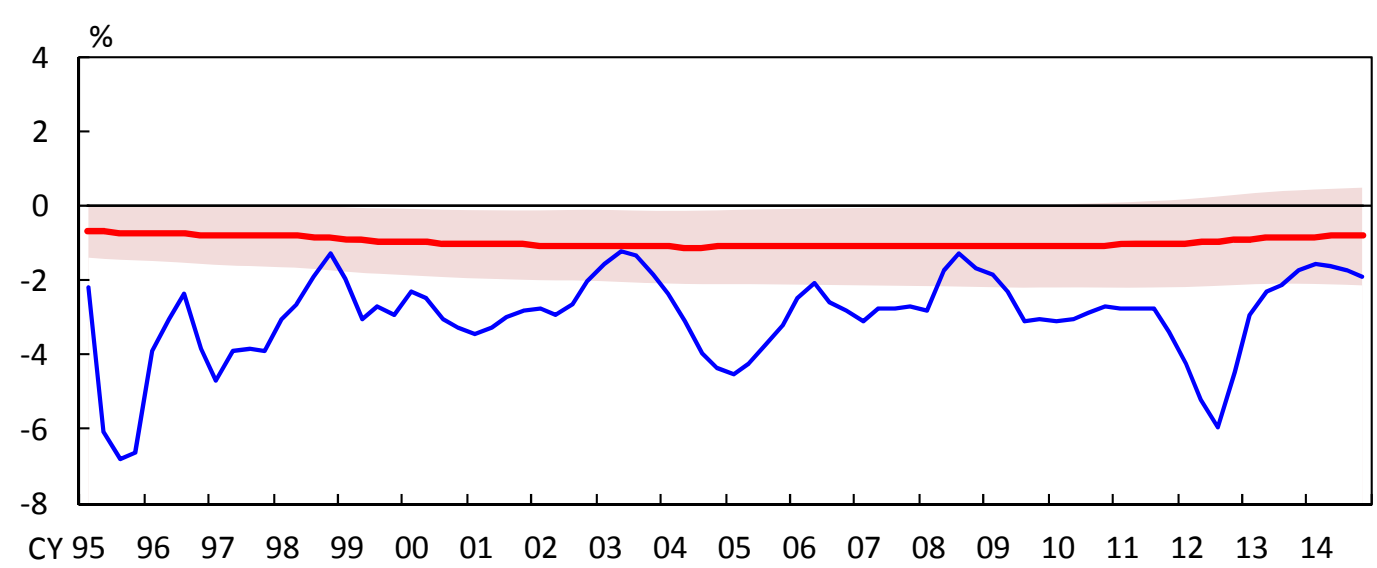

Humped $\uparrow$

Figure 6. Components of yield curves

The shaded areas indicate 90 percent confidence intervals. 
(a) Yields for all maturities

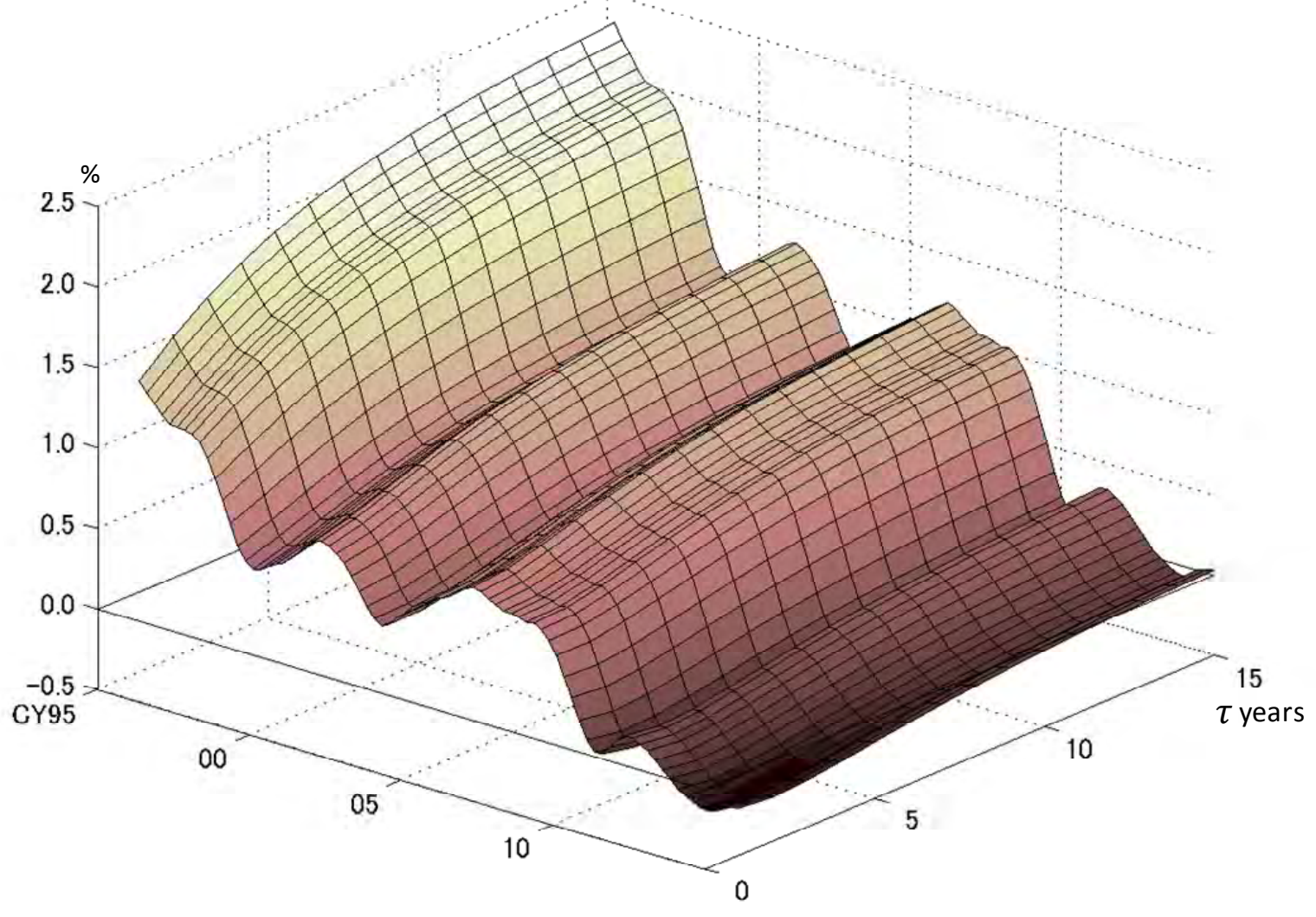

(b) Yields for selected maturities

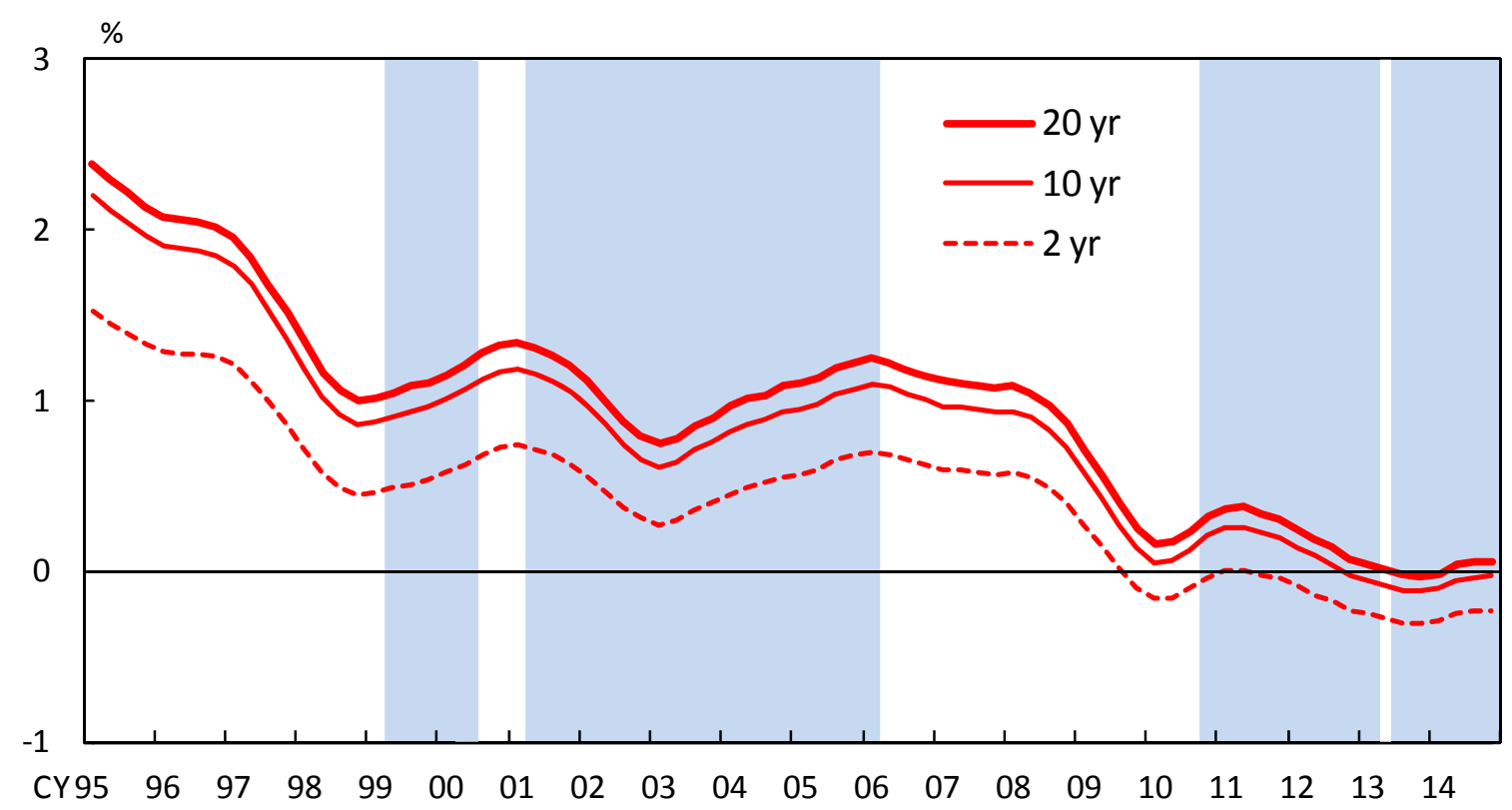

Figure 7. The natural yield curve

The shaded areas indicate, from left to right, the zero interest rate policy, quantitative easing, comprehensive monetary easing, and quantitative and qualitative monetary easing. 
(a) Zero interest rate policy

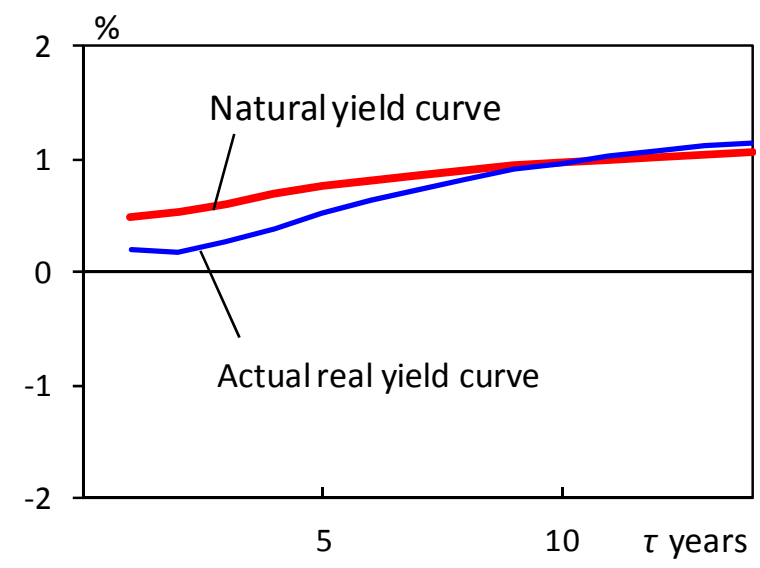

(c) Comprehensive monetary easing

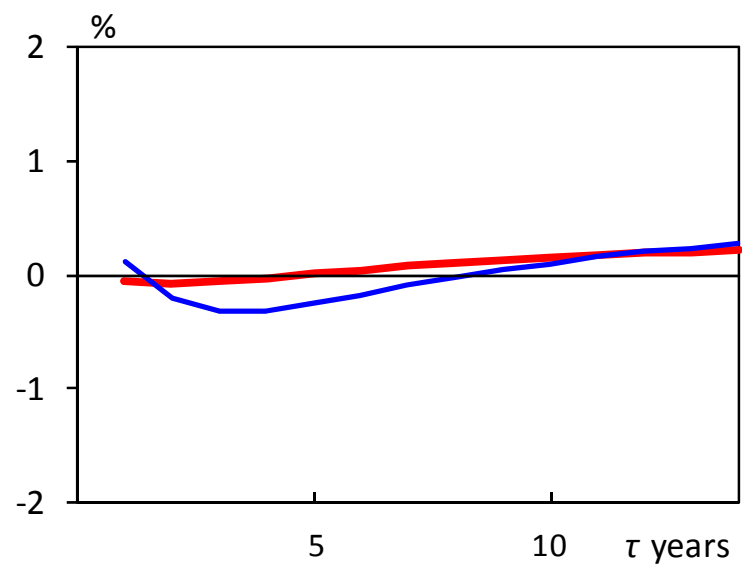

(b) Quantitative easing

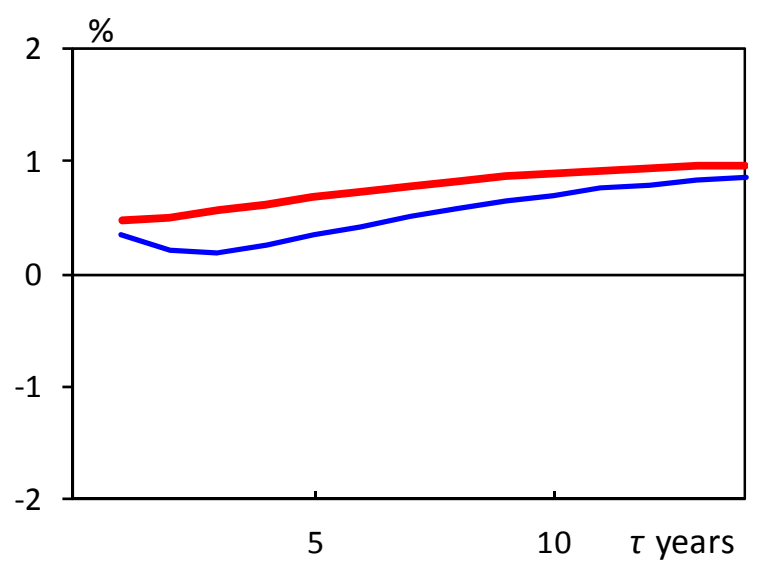

(d) QQE

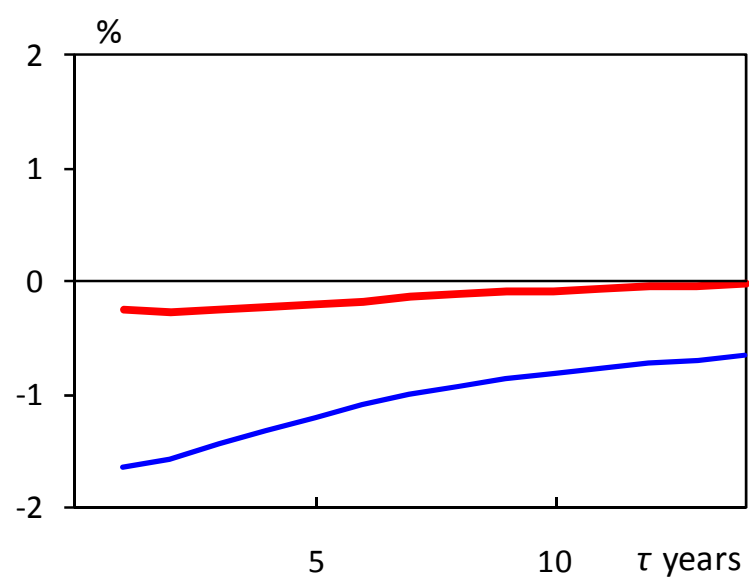

Figure 8. Yield curves during each of the monetary easing programs 
(a) 10-year maturity

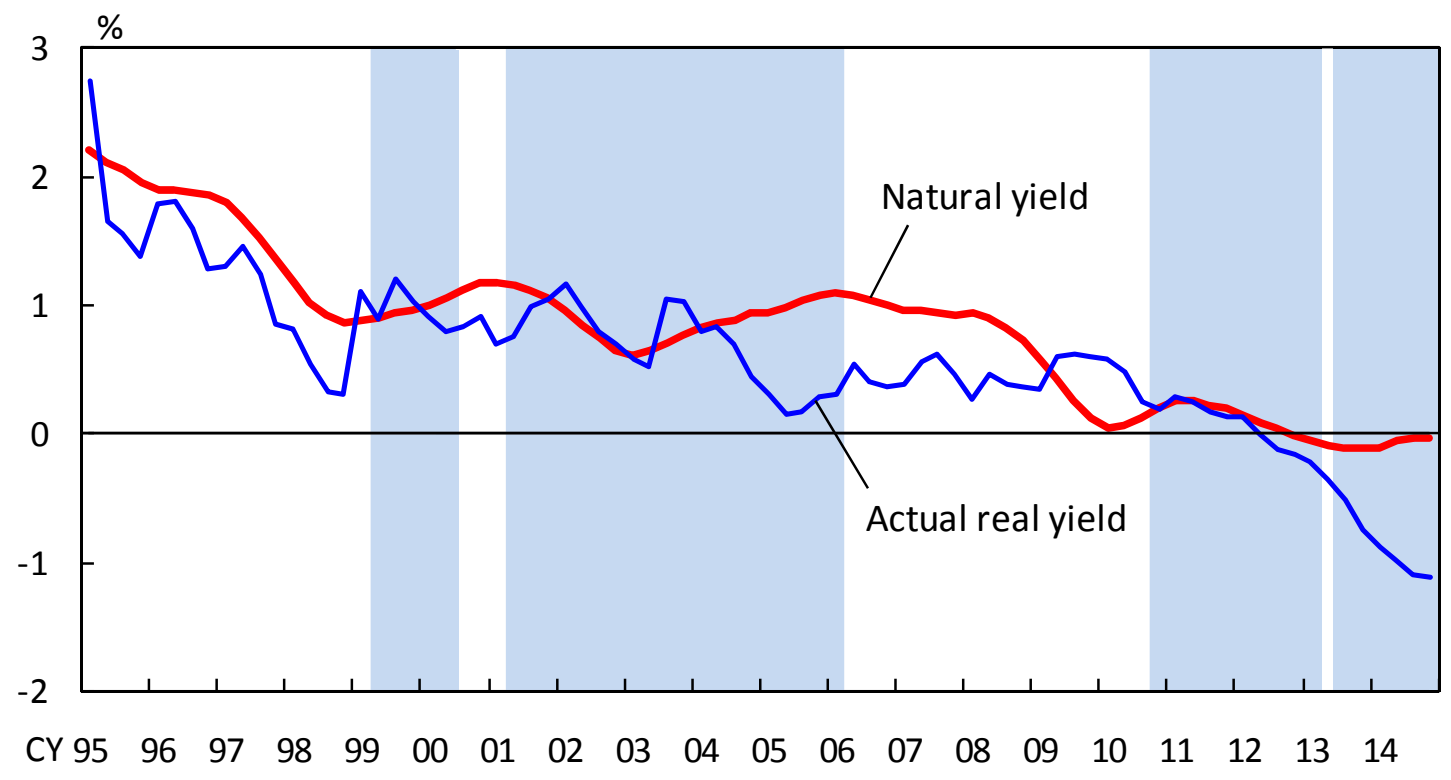

(b) 2-year maturity

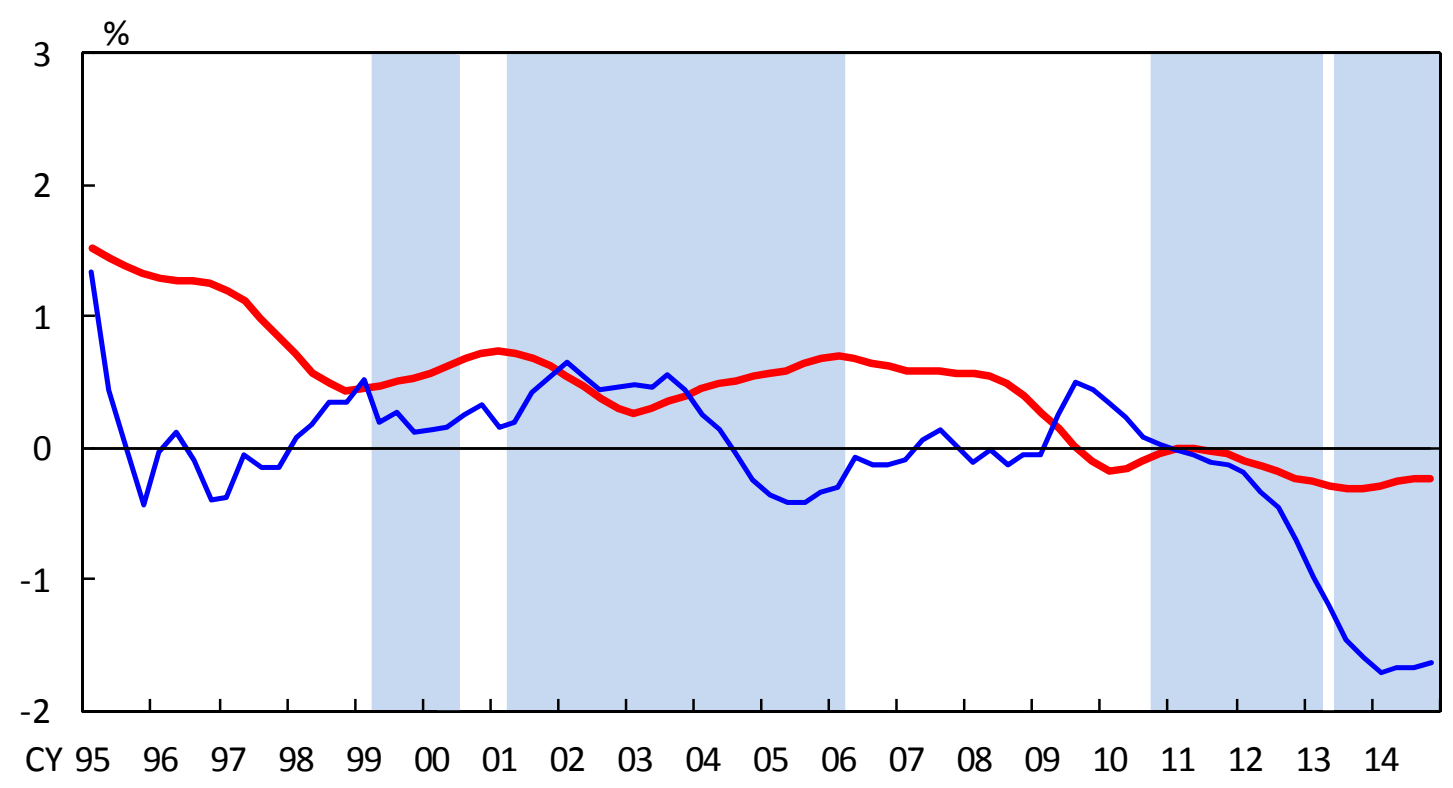

Figure 9. The natural yields and actual real yields

The shaded areas indicate, from left to right, the zero interest rate policy, quantitative easing, comprehensive monetary easing, and quantitative and qualitative monetary easing. 


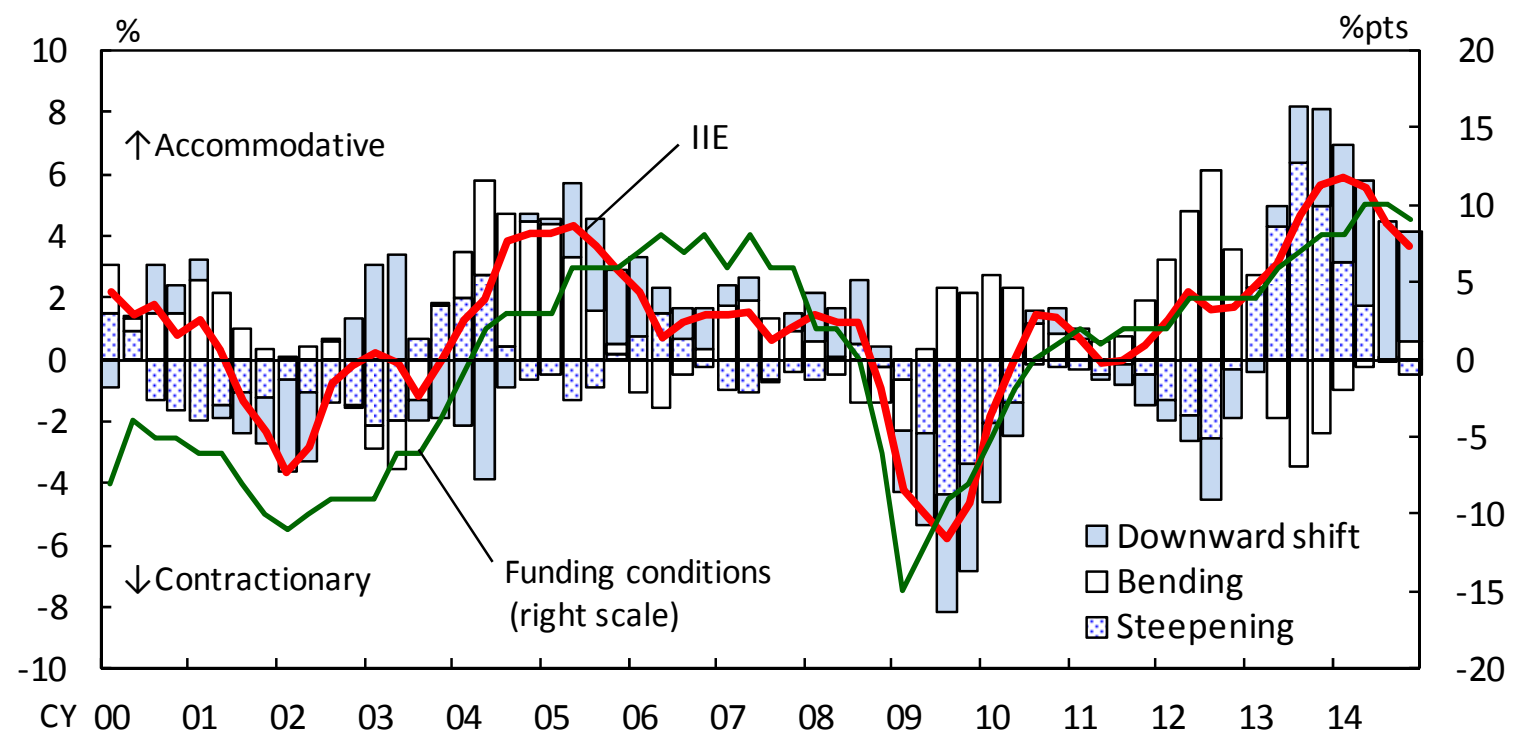

Figure 10. The IIE and firms' funding conditions

The figure shows the 4-quarter moving average of the IIE. Firms' funding conditions are represented by the D.I. for firms' financial position, which is obtained from the Tankan, Short-term Economic Survey of Enterprises in Japan, reported by the Bank of Japan. 